Research Article

\title{
Existence and Uniqueness Results for Two-Term Nonlinear Fractional Differential Equations via a Fixed Point Technique
}

\author{
H. R. Marasi $\mathbb{i D}^{1}$ and H. Aydi $\mathbb{D}^{2,3,4,5,6}$ \\ ${ }^{1}$ Department of Applied Mathematics, Faculty of Mathematical Sciences, University of Tabriz, Tabriz, Iran \\ ${ }^{2}$ Nonlinear Analysis Research Group, Ton Duc Thang University, Ho Chi Minh City, Vietnam \\ ${ }^{3}$ Faculty of Mathematics and Statistics, Ton Duc Thang University, Ho Chi Minh City, Vietnam \\ ${ }^{4}$ Department of Medical Research, China Medical University, Taichung 40402, Taiwan \\ ${ }^{5}$ Department of Mathematics and Applied Mathematics, Sefako Makgatho Health Sciences University, \\ Ga-Rankuwa, South Africa \\ ${ }^{6}$ Université de Sousse, Institut Supérieur d'Informatique et des Techniques de Communication, \\ H. Sousse 4000, Tunisia \\ Correspondence should be addressed to H. R. Marasi; marasi@tabrizu.ac.ir and H. Aydi; hassen.aydi@tdtu.edu.vn
}

Received 3 December 2020; Revised 9 December 2020; Accepted 16 December 2020; Published 11 January 2021

Academic Editor: Naeem Saleem

Copyright (c) 2021 H. R. Marasi and H. Aydi. This is an open access article distributed under the Creative Commons Attribution License, which permits unrestricted use, distribution, and reproduction in any medium, provided the original work is properly cited.

The work addressed in this paper is to ensure the existence and uniqueness of positive solutions for initial value problems for nonlinear fractional differential equations with two terms of fractional orders. By virtue of recent fixed point theorems on mixed monotone operators, we get some new straightforward results with a wide range of applications.

\section{Introduction}

In recent years, we see a great interest to consider fractional differential equations in various kinds of studies. Namely, these equations have striking applications in diverse science. Moreover, during the past decades, new areas for applications of fractional differential and integral equations with initial boundary conditions have been appeared. In the modeling of significant numbers of phenomena occurring in engineering and scientific subjects, we encountered a problem of such kinds. In multiple cases, modeling by fractional operators can also be done better than modeling by ordinary derivative operators. One can see fractional operators in applications of rheology, control, viscoelasticity, porus structures, chemical physics, electrochemistry, and significant number of other branches of science (see [1-3] for more details). For these reasons, there exist many articles about the existence and uniqueness of solutions of problems containing operators of fractional orders and using fixed point techniques [4-27]. But, the number of research studies on the study of existence and uniqueness of solutions for FDEs with two or more terms of fractional operators is limited. Fujita [28] studied the following specific Cauchy problem:

$$
\frac{\partial^{\alpha_{1}}}{\partial t} w(t, x)=\frac{\partial^{\alpha_{2}}}{\partial t} w(t, x), \quad 1 \leq \alpha_{1}, \alpha_{2} \leq 2
$$

and established some existence and uniqueness results. In [29], the author considered an initial value problem of the following form:

$$
\begin{aligned}
& D^{\alpha} y(t)=g\left(t, D^{\beta} y(t)\right), \quad 0<t \leq 1, \\
& y^{(k)}(0)=\mu_{k}, \quad 0 \leq k \leq n-1, k \in Z,
\end{aligned}
$$

where $n-1<\beta<\alpha<n$, and studied existence and uniqueness of related solutions. Based on the results of the above paper, the following exacting conditions should be satisfied on $g$ for the existence of solutions: 
(C1) $g$ is a continuous and differentiable function on $[0,1] \times \mathbf{R} \longrightarrow \mathbf{R}$.

(C2) For $t \in I, g(t, 0) \neq 0$ and $g(0,0)=0$, where $I$ is any compact subinterval of $[0,1]$.

(C3) There exists a continuous function $q$ which satisfies $C \sup _{t \in[0,1]} \int_{0}^{t}(t-r)^{\alpha-\beta-1} q(r) \mathrm{d} r<1, \quad(n-1<\beta<\alpha<n)$

where $C=1 / \Gamma(\alpha-\beta)$.

To detect a unique solution other than (C1)-(C3), the following restriction should hold.

(C4) Let $w_{1}$ and $w_{2}$ be any real numbers and $q \in C([0,1],[0, \infty))$ be the continuous function appeared in (4), then

$$
\left|g\left(t, w_{1}\right)-g\left(t, w_{2}\right)\right| \leq q(t)\left|w_{1}-w_{2}\right|, \quad 0 \leq t \leq 1 .
$$

The authors in $[16,30]$ have studied the same problem. In [16], we observe that the necessary conditions to exist at least one solution for (2) and (3) are (C1), (C2), and the following condition.

(C5) For a continuous function $y \in C[0, \infty)$, there exist positive numbers $c$ and $d$ such that

$$
|g(t, y(t))| \leq c|y(t)|+d, \quad 0 \leq t \leq 1
$$

Also, the additional condition that $g(t, x(t))$ satisfies a Lipschitz condition is needed to prove the uniqueness of the solution:

$$
\left|g\left(t, z_{1}(t)\right)-g\left(t, z_{2}(t)\right)\right| \leq M\left|z_{1}(t)-z_{2}(t)\right|, \quad 0 \leq t \leq 1,
$$

where $M$ is the Lipschitz constant. In [31], the following general form is considered:

$$
\begin{aligned}
& D^{\alpha} y(t)=g\left(t, y(t), D^{\beta} y(t)\right), \quad 0<t \leq 1, \\
& y^{(k)}(0)=\mu_{k}, \quad 0 \leq k \leq n-1, k \in Z,
\end{aligned}
$$

where $n-1<\beta<\alpha<n,(n \in \mathbf{N})$ and $D^{\alpha}$ and $D^{\beta}$ are the fractional derivative orders in the Caputo definition and $g \in C([0,1] \times \mathbf{R})$. We have used an index fixed point theorem and obtained some new results about existence and also multiplicity of solutions. In this paper, we consider the same general form (8) and (9) and try to use new fixed point theorems proved for operators having mixed monotone property to establish new results with a wide range of applications for existence and uniqueness.

\section{Preliminaries}

For convenience of readers, we provide some useful definitions and previous results which we use throughout the paper.

, Definition 1 (see $[1,3])$. Let $\alpha>0$. The operator defined by

$$
I^{\alpha} y(t)=\int_{0}^{t} \frac{1}{(t-r)^{\alpha-1} \Gamma(\alpha)} y(r) \mathrm{d} r
$$

where $y \in C[0, \infty) \cap L_{\text {loc }}^{1}[0, \infty), \quad \alpha \in(m-1, m)$ and $m \in \mathbf{N}$, is the fractional integral of $y$ of order $\alpha$ in Riemann-Liouville definition.

After introducing the fractional integral operator, it is time to remind the fractional differentiation definitions. There are several ways to define fractional derivative. Here, we focus on the Caputo definition.

Definition 2 (see $[1,3]$ ). Let $n=[\alpha]+1$ where $\alpha \geq 0$. If $y(t) \in A C^{n}[0,1]$, then the fractional derivative of $y$ in Caputo sense exists on the interval $[0,1]$, almost everywhere, and is obtained by

$$
{ }^{c} D^{\alpha} y(t)=\int_{0}^{t} \frac{y^{n}(r)}{\Gamma(n-\alpha)(t-r)^{\alpha-n+1}} \mathrm{~d} r .
$$

For the sake of convenience, here, we mention some properties of Caputo fractional operator. For the power function $f(t)=t^{q}, q \geq 0$, taking the Caputo derivative yields

$$
{ }^{c} D^{\alpha} t^{q}= \begin{cases}\frac{\Gamma(q+1)}{\Gamma(q-\alpha+1)} t^{q-\alpha}, & (q \geq n-1), \\ 0, & (q \leq n-1) .\end{cases}
$$

By virtue of this formula, one can deduce ${ }^{c} D^{\alpha} k=k^{c} D^{\alpha} t^{0}=0$. Therefore, unlike Riemann-Liouville derivative, the Caputo derivative of a constant function is zero. In the following equality:

$$
{ }^{c} D^{\alpha}\left(a_{0} t^{r}+a_{1} t^{r-1}+\cdots+a_{1}\right)=0, \quad \alpha \in(m-1, m),
$$

the polynomial's degree is less than or equal to $m-1$, i. e, $r \leq m-1$. From the following relation, it is clear that similar to the case of integer order, the $\alpha$ th fractional integral of the Caputo fractional derivative of order $\alpha$ requires to know the values of the function and its integer order derivatives:

$$
I^{\alpha} D^{\alpha} g(t)=g(t)-\sum_{l=0}^{m-1} g^{l}\left(t_{0}^{+}\right) \frac{t^{l}}{l !}, \quad m-1<\alpha \leq m .
$$

The results of the paper about the existence and uniqueness are expressed by the famous beta function given as follows.

Definition 3. The following integral defines the beta function: 


$$
B(x, y)=\int_{0}^{1} s^{x-1}(1-s)^{y-1} \mathrm{~d} s, \quad x, y>0 .
$$

It is known that

$$
B(r, s)=\frac{\Gamma(r) \Gamma(s)}{\Gamma(r+s)},
$$

where $\Gamma$ denotes the Gamma function.

The following lemma transforms problems (8) and (9) to an integral equation $[29,30]$, at which the nonlinear term $g$ needs to satisfy the conditions (C1) and (C2).

Lemma 1 (see [29]). Let $n \in \mathbf{N}, \beta<\alpha$ and $\alpha, \beta \in(n-1, n)$. If (C1) and (C2) hold, then $y \in C^{n}[0,1]$ is a solution of the following problem:

$$
\begin{aligned}
& D^{\alpha} y(x)=g\left(x, y(x), D^{\beta} y(x)\right), \quad 0<x \leq 1, \\
& y^{(k)}(0)=\mu_{k}, \quad 0 \leq k \leq n-1, k \in Z,
\end{aligned}
$$

if and only if

$$
y(x)=\sum_{k=0}^{n-1} \frac{x^{k}}{k !} \eta_{k}+\frac{1}{\Gamma(\beta)} \int_{0}^{x}(x-r)^{\beta-1} w(r) \mathrm{d} r, \quad 0 \leq x \leq 1,
$$

where $w$ is a continuous function and satisfies the following integral equation:

$$
w(x)=\int_{0}^{1} K(x, r) g(r, w(r)) \mathrm{d} r, \quad 0 \leq x \leq 1,
$$

where

$$
K(x, r)=\frac{1}{\Gamma(\alpha-\beta)}(x-r)_{+}^{\alpha-\beta-1}
$$

with

$$
(x-r)_{+}^{\alpha-\beta-1}= \begin{cases}(x-r)^{\alpha-\beta-1}, & r \leq x, r, x \in[0,1] \\ 0, & r \geq x .\end{cases}
$$

Here and anywhere below, the function $K(x, r)$ is the Green function.

Lemma 2. Let $n, m \in \mathbf{N}$ and $n-1<\beta \leq m-1<\alpha<m$. If (C1) and (C2) hold, then $y \in C^{m}[0,1]$ is a solution of (17) and (18) if and only if

$$
y(x)=\sum_{l=0}^{n-1} \frac{x^{l}}{l !} \eta_{l}+\int_{0}^{x} \frac{(x-r)^{n-1} w(r) \mathrm{d} r}{(n-1) !}, \quad 0 \leq x \leq 1,
$$

where $w \in C[0,1]$ satisfies the following integral equation:

$$
\begin{aligned}
w(x)= & \sum_{l=0}^{m-n-1} \frac{x^{l}}{l !} \eta_{n+l}+\frac{1}{\Gamma(\alpha-n)} \int_{0}^{x}(x-r)^{\alpha-n-1} \\
& \times f\left(r, w(r), \frac{1}{\Gamma(n-\beta)}\right. \\
& \left.\int_{0}^{r}(r-h)^{n-\beta-1} w(h) \mathrm{d} h\right) \mathrm{d} r, \quad 0 \leq x \leq 1 .
\end{aligned}
$$

Now, we present the following lemma which will be useful in proving an existence and uniqueness theorem of the solution of (8) and (9).

Lemma 3. For the Green function $G(t, s)$, the following relations hold:

$$
\begin{aligned}
& \int_{0}^{1} K(x, r) \mathrm{d} r \leq B(\alpha-\beta, \alpha-\beta), \quad 0 \leq x \leq 1, \\
& \int_{0}^{1} K(x, r) \mathrm{d} r \geq \frac{\Gamma(\alpha-\beta)}{\Gamma 2(\alpha-\beta)}, \quad 0 \leq x \leq 1 .
\end{aligned}
$$

Proof. By making use of (14), it is easy and straightforward to prove the properties for $K(x, r)$.

Now, we provide some definitions and a fixed point theorem involving mixed monotone operators. We suggest references $[19,20]$ for more details.

Definition 4. Let $(E,\|\|$.$) be a Banach space and P \subseteq E$ be a nonempty closed convex subset. $P$ is a cone in $E$ if

(I) For any $x \in P$ and $\lambda \geq 0$, then $\lambda x \in P$

(II) For any $x \in P$, if $-x \in P$ then $x=\theta$

where $\theta$ denotes the zero element of the Banach space $E$.

If there exists a constant $C>0$ such that, for any $x, y \in P$ with $\theta \leq x \leq y$, we have $\|x\| \leq C\|y\|$, then $P$ is called a normal cone. Then, we give the definition of a partially ordered Banach space. We say that $E$ is partially ordered by $P$ if $x \leq y$ iff $y-x \in P$, for any $x, y \in P$. For arbitrary $x_{1}, x_{2} \in E$, the ordered interval is defined by $\left[x_{1}, x_{2}\right]=\{x \in E$ $\left.\mid x_{1} t \leq n x q \leq h x_{2}\right\}$. Let $T: E \longrightarrow E$ be an operator. If $u \leq v$ implies $T u \leq T v$, then $T$ is called increasing.

Let us briefly recall that the operator $T: P \times P \longrightarrow P$ is increasing in its first variable and decreasing in its second variable, if from $x_{i}, y_{i} \in P(i=1,2), x_{1} \leq x_{2}, y_{1} \geq y_{2}$, one can deduce $T\left(x_{1}, y_{1}\right) \leq T\left(x_{2}, y_{2}\right)$. If $x \geq \theta$ implies that $T(x) \geq \theta$, then the operator $T$ is called positive.

In the following definition, $P$ is a cone on the Banach space $(E,\|\cdot\|)$.

Definition 5 (see $[19,20]$ ). Suppose $T: P \times P \longrightarrow P$ is increasing in its first variable and decreasing in its second variable, then $T$ is called a mixed monotone operator.

If for an element $x \in P, T(x, x)=x$, then $x$ is called a fixed point of $T$.

Theorem 1 (see [4]). Suppose T: $P \times P \longrightarrow P$ is a mixed monotone operator and let the following conditions hold:

(i) If $c \in(0,1), x, y \in P$, then there exists $\alpha(c, x, y) \in(1, \infty)$ such that

$$
T(c x, y) \leq c^{\alpha(c, x, y)} T(x, y) .
$$

(ii) There exist two elements $u_{0}, v_{0} \in P, r \in(0,1)$ such that 


$$
\begin{aligned}
u_{0} & \leq r v_{0} \\
T\left(u_{0}, v_{0}\right) & \geq u_{0} \\
T\left(v_{0}, u_{0}\right) & \leq v_{0} .
\end{aligned}
$$

Then, $T$ has a unique fixed point $u^{*}$ in $\left[u_{0}, r v_{0}\right]$ such that $T\left(u^{*}, u^{*}\right)=u^{*}$. Moreover, one can construct successively the following iterates:

$$
\begin{aligned}
& u_{n}=T\left(u_{n-1}, v_{n-1}\right), \\
& v_{n}=T\left(v_{n-1}, u_{n-1}\right), \quad n=1,2, \ldots,
\end{aligned}
$$

starting from $x_{0}, y_{0} \in\left[u_{0}, r v_{0}\right]$ to get the fixed point of $T$. Also, $\left\|u_{n}-u^{*}\right\| \longrightarrow 0,\left\|v_{n}-u^{*}\right\| \longrightarrow 0$ as $n \longrightarrow \infty$.

\section{Main Results}

In this section, we prove new existence and uniqueness results for nonlinear fractional differential equations with two terms of fractional derivatives. Compared with the results of the previous studies, our results are simple and straightforward. They are expressed with the values of beta and gamma functions. For these reasons, these results can be applied for a wide range of problems. To make effective our obtained result, some examples provided at the end of section cannot be considered by techniques of $[16,30]$. In order to apply the fixed point results concerning the mixed monotone operators for the study of (8) and (9), we first consider $E=C([0,1])$ as a suitable Banach space, and let the norm on $E$ be defined by

$$
\|y\|=\max \{|y(t)|, \quad 0 \leq t \leq 1\} .
$$

Let us consider

$$
P=\left\{y \in E \mid \min y(t) \geq 0, \quad 0 \leq t \leq 1,{ }^{C} D^{\alpha} \text { is positive }\right\} .
$$

It is obvious that $P$ is a normal cone in $E$.

In the following theorem, $g$ is the function appeared in the right side of equation (8).

\section{Theorem 2. Assume that}

(C6) $g(t, u, v)$ is a continuous function which is increasing in its first variable $u$ and decreasing in its second variable $v$.

(C7) For any $\eta \in(0,1)$, let $u$ and $v$ be two arbitrary elements of $P$ so that there exists $\lambda(\eta, u, v) \in(1, \infty)$ such that

$$
g(t, \eta u, v) \leq \eta^{\lambda(\eta, u, v)} g(t, u, v)
$$

(C8) Let $u_{0}$ and $v_{0}$ be two elements of $P$ and $\mu \in(0,1)$ such that

$$
\begin{gathered}
u_{0}(t) \leq \mu v_{0}(t), \\
g\left(t, u_{0}, D^{\beta} v_{0}\right) \geq \frac{u_{0} \Gamma[2(\alpha-\beta)]}{\Gamma(\alpha-\beta)}, \\
g\left(t, u_{0}, D^{\beta} v_{0}\right) \leq \frac{v_{0}}{B(\alpha-\beta, \alpha-\beta)} .
\end{gathered}
$$

Then, for equation (8) with initial conditions (9), we have a unique solution $u^{*} \in\left[u_{0}, \mu v_{0}\right]$. Furthermore, by computing the iterates,

$$
\begin{aligned}
& u_{n+1}(t)=\int_{0}^{t} K(t, r) g\left(r, u_{n}(r), \frac{\partial}{\partial r} v_{n}(r)\right) \mathrm{d} r \\
& v_{n+1}(t)=\int_{0}^{t} K(t, r) g\left(r, v_{n}(r), \frac{\partial}{\partial r} u_{n}(r)\right) \mathrm{d} r .
\end{aligned}
$$

For $n=0,1, \ldots$, one can find the unique solution. In other words, $\left\|u_{n}-u^{*}\right\| \longrightarrow 0,\left\|v_{n}-u^{*}\right\| \longrightarrow 0$ as $n \longrightarrow \infty$.

Proof. First, we define the operator $T$ as follows:

$$
T(u, v)(t)=\int_{0}^{t} K(t, r) g\left(r, u(r), D^{\beta} v(r)\right) d r,
$$

where $K(t, r)$ is the Green function (21). Taking the definition of the operator $T$ into account and Lemma 3 , it is clear that a certain function $u(t)$ is a solution of (2) and (3) iff $u(t)$ is a fixed point of $T$, that is, $T(u, u)=u$. Hence, it will be sufficient to show that $T$ satisfies the conditions of Theorem 1. By virtue of (C6), it immediately follows that $T$ is increasing in its first variable $u$ and decreasing in its second variable $v$. On the other hand, under the hypothesis of (C7), if $\eta \in(0,1)$ and $u, v$ are two elements of $P$, then there exists $\lambda(\eta, u, v) \in(1, \infty)$ such that

$$
\begin{aligned}
T(\eta u(t), v(t)) & =\int_{0}^{t} K(t, r) g\left(r, \eta u(r), \frac{\partial}{\partial r} v(r)\right) \mathrm{d} r \\
& \leq \eta^{\alpha} \int_{0}^{t} K(t, r) g\left(r, u(r), \frac{\partial}{\partial r} v(r)\right) \mathrm{d} r \\
& =\eta^{\alpha} T(u(t), v(t)) .
\end{aligned}
$$

Also, with the help of (C8), and using Lemma 3, we can choose $u_{0}$ and $v_{0}$ such that

$$
\begin{aligned}
& T\left(u_{0}(t), v_{0}(t)\right)=\int_{0}^{t} K(t, r) g\left(r, u_{0}(r), \frac{\partial}{\partial r} v_{0}(r)\right) \mathrm{d} r \leq u_{0}, \\
& T\left(v_{0}(t), u_{0}(t)\right)=\int_{0}^{t} K(t, r) g\left(r, v_{0}(r), \frac{\partial}{\partial r} u_{0}(r)\right) \mathrm{d} r \geq v_{0} .
\end{aligned}
$$

Consequently, it is obvious that all essentials of Theorem 1 are provided by (C6)-(C8), and therefore, there is a unique 
positive solution $\left(u^{*}, u^{*}\right)$ so that $T\left(u^{*}, u^{*}\right)=u^{*}$. It is the unique solution of (8) and (9). The proof is complete.

Example 1. Let us consider the following set of nonlinear differential equations of fractional order

$$
{ }^{c} D^{m+(3 / 4)} y(t)=\psi(t)-\left[{ }^{c} D^{m+(1 / 4)} y(t)\right]^{2}, \quad 0<t \leq 1,
$$

subject to

$$
y^{l}(0)=0, \quad l=0,1,2, \ldots, m,
$$

where $m$ is a positive integer, $\psi(t)$ is a continuous function such that (HTML translation failed), and $\eta_{1}=\sqrt{\pi} \leq \psi(t) \leq m \pi=\eta_{2}$. In connection with Theorem 2, set $\alpha=m+(3 / 4)$ and $\beta=m+(1 / 4)$. By virtue of this theorem and Lemma 3 , the existence of a unique solution for problems (37) and (38) is equivalent to a unique solution for the following integral equation:

$$
y(t)=\int_{0}^{t} K(t, r)\left(\psi(r)-\left[{ }^{c} D^{m+(1 / 4)} y(r)\right]^{2}\right) \mathrm{d} r
$$

where with the help of Lemma 3, one can deduce $L_{1}=1 / \sqrt{\pi} \leq \int_{0}^{1} K(t, r) \mathrm{d} r \leq 2 / \pi=L_{2}$. We note that if $u, v \in P$, defined by (30), then $g(t, u, v)=\psi(t)-\left[{ }^{c} D^{m+(1 / 4)} v(t)\right]^{2}$ is increasing in $u$ and decreasing in $v$. Now, let us define $A(u, v)=\int_{0}^{t} K(t, r)\left(\psi(r)-\left[{ }^{c} D^{m+(1 / 4)} v(r)\right]^{2}\right) \mathrm{d} r$ and choose $u_{0}=1, v_{0}=2 m$. Then, considering that $u_{0}, v_{0}$ are constant, we can show that

$$
\begin{gathered}
u_{0} \leq \frac{1}{2} v_{0}, \\
A\left(u_{0}, v_{0}\right) \geq L_{1} \eta_{1} \geq 1=u_{0}, \\
A\left(v_{0}, u_{0}\right) \leq \frac{2}{\pi} \eta_{2} \leq 2 m=v_{0} .
\end{gathered}
$$

Therefore, using Theorem 2, we conclude that problems (37) and (38) possess a unique positive solution such that $1 \leq y(t) \leq m$.

Example 2. In this example, we find a unique positive solution for the nonlinear fractional initial value problems of the following form:

$$
{ }^{c} D^{n+(2 / 3)} y(t)=\chi(t)-\frac{1}{y(t)}-\sqrt{{ }^{c} D^{n+(2 / 3)} y(t)}, \quad 0<t \leq 1,
$$

$$
y^{l}(0)=0, \quad l=0,1,2, \ldots, n,
$$

where $n \in Z^{+}, \chi(t)$ is a continuous function such that $\chi(0) \neq 0$, and $\tau_{1}=315 / 100 \leq \chi(t) \leq 945 / 100=\tau_{2}$. In connection with Theorem 2 , set $\alpha=n+(2 / 3)$ and $\beta=n+(1 / 3)$. By virtue of this theorem and Lemma 3, the existence of a unique solution for problems (41) and (42) is equivalent to a unique solution for the following integral equation:

$$
y(t)=\int_{0}^{t} K(t, r)\left[\chi(r)-\frac{1}{y(r)}-\sqrt{{ }^{c} D^{n+(1 / 3)}} y(r)\right] \mathrm{d} r
$$

where with the help of Lemma 3, one deduces $2 \leq \int_{0}^{1} K(t, r) \mathrm{d} r \leq 53 / 10$. We note that if $u, v \in P$ are defined by (30), then $g(t, u, v)=\chi(s)-1 / u(t)-\sqrt{{ }^{c} D^{n+(1 / 3)} v(t)}$ is increasing in $u$ and decreasing in $v$.

For $c \in(0,1)$, consider $1<\alpha(c, u, v)<2$; then, we have

$$
g(t, c u(t), v(t))=\chi(t)-\frac{1}{c u(t)}-\sqrt{{ }^{c} D^{n+(1 / 3)} v(t)} \leq c^{\alpha}\left[\chi(t)-\frac{1}{u(t)}-\sqrt{{ }^{c} D^{n+(1 / 3)} v(t)}\right]=c^{\alpha} g(t, u(t), v(t)) .
$$

Now, let us define $A(u, v)=\int_{0}^{t} K(t, r)[\chi(r)-1 / u(r)-$ $\left.\sqrt{{ }^{c} D^{n+(1 / 3)} v(r)}\right] \mathrm{d} r$ and choose $u_{0}=53 / 10, v_{0}=51$. Then, regarding that $u_{0}, v_{0}$ are constant functions, we get

$$
\begin{aligned}
& A\left(u_{0}, v_{0}\right)=\int_{0}^{1} K(t, r)\left[\chi(r)-\frac{1}{u_{0}}-\sqrt{{ }^{c} D^{n+(1 / 3)} v_{0}}\right] \mathrm{d} r \geq 2 \gamma_{1}-1 \geq \frac{53}{10}=u_{0}, \\
& A\left(v_{0}, u_{0}\right)=\int_{0}^{1} K(t, r)\left[\chi(r)-\frac{1}{v_{0}}-\sqrt{{ }^{c} D^{n+(1 / 3)} u_{0}}\right] \mathrm{d} r \leq \frac{53}{10} \gamma_{-} \frac{2}{v_{0}} \leq 51=v_{0} .
\end{aligned}
$$


Therefore, using Theorem 2, we conclude that for problems (38)-(41), there is a unique positive solution such that $y(t) \in[53 / 10, t 51]$ for each $m$.

\section{Conclusion}

In this research, we considered nonlinear fractional differential equations of arbitrary order with two terms of fractional orders. By virtue of recent fixed point theorems on normal cones for mixed monotone operators, we obtained some new results for existence and uniqueness of solutions of these equations involving initial conditions, which are more applicable compared with previous existing results. The previous results can be proved based on very strict conditions on the function $g$. While, in our paper, the function $g\left(t, u, D^{\alpha} u\right) \equiv g(t, u, v)$ needs to be increasing in its first variable $u$ and decreasing in its second variable $v$. Also, establishing the conditions (C7) and (C8) is not difficult. On the other hand, our results provided a constructive approach, based on an iterative relation, to find the solution. Indeed, the interval $(0,1]$ can be generalized to $(0, T]$. The presented examples, at the end of the paper, cannot be considered by the previous works.

\section{Data Availability}

No data were used to support this study.

\section{Conflicts of Interest}

The authors declare no conflicts of interest.

\section{Authors' Contributions}

All authors contributed equally and significantly in writing this article.

\section{References}

[1] A. A. Kilbas, H. M. Srivastava, and J. J. Trujillo, "Theory and applications of fractional differential equations," North-Holland Mathematics Studies, vol. 204, pp. 7-10, 2006.

[2] K. S. Miller and B. Ross, An Introduction to the Fractional Calculus and Fractional Differential Equations, Wiley, New York, NY, USA, 1993.

[3] I. Podlubny, Fractional Differential Equations, Academic Press, New York, NY, USA, 1999.

[4] C. B. Zhai, "Fixed point theorems for a class of mixed monotone operators with convexity," Fixed Point Theory Application, vol. 2013, 119 pages, 2013.

[5] H. R. Marasi, H. Afshari, and C. B. Zhai, "Some existence and uniqueness results for nonlinear fractional partial differential equations," Rocky Mountain Journal of Mathematics, vol. 47, no. 2, pp. 571-585, 2017.

[6] H. Afshari, H. Marasi, and H. Aydi, "Existence and uniqueness of positive solutions for boundary value problems of fractional differential equations," Filomat, vol. 31, no. 9, pp. 2675-2682, 2017.

[7] H. R. Marasi, H. Afshari, M. Daneshbastam, and C. B. Zhai, "Fixed points of mixed monotone operators for existence and uniqueness of nonlinear fractional differential equations,"
Journal of Contemporary Mathematical Analysis, vol. 52, no. 1, pp. 78-84, 2017.

[8] H. Aydi, A. Felhi, H. Afshari, and H. R. Marasi, "Fixed point results for couplings on abstract metric spaces and an application," Fixed point results for couplings on abstract metric spaces and an application, vol. 9, no. 2, pp. 57-67, 2018.

[9] C. B. Zhai, L. Zhao, S. Li, and H. R. Marasi, "New existing results for a system of nonlinear third-order differential equation via fixed point index," Journal of Applied Analysis and Computation, vol. 8, no. 6, pp. 1694-1706, 2018.

[10] D. Baleanu, R. P. Agarwal, H. Mohammadi, and S. Rezapour, "Some existence results for a nonlinear fractional differential equation on partially ordered banach spaces," Boundary Value Problems, vol. 2013, no. 1, 2013.

[11] F. Chen, D. Baleanu, and G.-C. Wu, "Existence results of fractional differential equations with riesz-caputo derivative," The European Physical Journal Special Topics, vol. 226, no. 1618, pp. 3411-3425, 2017.

[12] H. Jafari, H. Jassim, M. Al Qurashi, and D. Baleanu, “On the existence and uniqueness of solutions for local fractional differential equations," Entropy, vol. 18, no. 11, p. 420, 2016.

[13] X. Zhang, L. Wang, and Q. Sun, "Existence of positive solutions for a class of nonlinear fractional differential equations with integral boundary conditions and a parameter," Applied Mathematics and Computation, vol. 226, pp. 708-718, 2014.

[14] S. Liang and J. Zhang, "Positive solutions for boundary value problems of nonlinear fractional differential equation," Nonlinear Analysis: Theory, Methods \& Applications, vol. 71, no. 11, pp. 5545-5550, 2009.

[15] C. B. Zhai, L. Zhao, S. Li, and H. R. Marasi, "On some properties of positive solutions for a third-order three-point boundary value problem with a parameter," Advances in Difference Equations, vol. 2017, p. 187, 2017.

[16] J. Deng and Z. Deng, "Existence of solutions of initial value problems for nonlinear fractional differential equations," Applied Mathematics Letters, vol. 32, pp. 6-12, 2014.

[17] D. Gou and V. Lakshmikantham, Nonlinear Problems in Abstract Cones, Academic Press, San Diego, CA, USA, 1988.

[18] H. Wong, C. J. Radke, and S. Morris, "The motion of long bubbles in polygonal capillaries. part 1. thin films," Journal of Fluid Mechanics, vol. 292, pp. 71-94, 1995.

[19] D. Guo and V. Lakshmikantham, "Coupled fixed points of nonlinear operators with applications," Nonlinear Analysis: Theory, Methods \& Applications, vol. 11, no. 5, pp. 623-632, 1987.

[20] D. Guo, "Fixed points of mixed monotone operators with applications," Applicable Analysis, vol. 31, no. 3, pp. 215-224, 1988.

[21] E. Ameer, H. Aydi, M. Arshad, H. Alsamir, and M. Noorani, "Hybrid multivalued type contraction mappings in $\alpha k$ complete partial b-metric spaces and applications," Symmetry, vol. 11, no. 1, p. 86, 2019.

[22] E. Ameer, H. Aydi, M. Arshad, and M. De la Sen, "Hybrid cirić type graphic -contraction mappings with applications to electric circuit and fractional differential equations," Symmetry, vol. 12, no. 3, p. 467, 2020.

[23] P. Patle, D. Patel, H. Aydi, and S. Radenović, "On H+type multivalued contractions and applications in symmetric and probabilistic spaces," Mathematics, vol. 7, no. 2, p. 144, 2019.

[24] A. Hussain, D. Baleanu, and M. Adeel, "Existence of solution and stability for the fractional order novel coronavirus (nCoV-2019) model," Advances in Difference Equations, vol. 2020, p. 384, 2020. 
[25] T. Kanwal, A. Hussain, H. Baghani, and M. De la Sen, "New fixed point theorems in orthogonal $F$ - metric spaces with application to fractional differential equation," Symmetry, vol. 12, no. 5, p. 832, 2020.

[26] N. Saleem, M. Abbas, B. Ali, and Z. Raza, "Fixed points of suzuki-type generalized multivalued $(f, \theta, L)$ - almost contractions with applications," Filomat, vol. 33, no. 2, pp. 499-518, 2019.

[27] N. Saleem, B. Ali, M. Abbas, and Z. Raza, "Fixed points of suzuki type generalized multivalued mappings in fuzzy metric spaces with applications," Fixed Point Theory Application, vol. 36, no. 1, pp. 1-18, 2015.

[28] Y. Fujita, "Cauchy problems of fractional order and stable processes," Japan Journal of Applied Mathematics, vol. 7, no. 3, pp. 459-476, 1990.

[29] N. Kosmatov, "Integral equations and initial value problems for nonlinear differential equations of fractional order," Nonlinear Analysis: Theory, Methods \& Applications, vol. 70, no. 7, pp. 2521-2529, 2009.

[30] J. Deng and L. Ma, "Existence and uniqueness of solutions of initial value problems for nonlinear fractional differential equations," Applied Mathematics Letters, vol. 23, no. 6, pp. 676-680, 2010.

[31] H. R. Marasi, H. Piri, and H. Aydi, "Existence and multiplicity of solutions for nonlinear fractional differential equations," Journal of Nonlinear Sciences and Applications, vol. 9, pp. 4639-4646, 2016. 